Public Health, Faculty of Veterinary Medicine, University of Calgary; ${ }^{4}$ Department of Pediatrics, Faculty of Medicine, University of Calgary

10.1136/jech-2014-205217.11

Introduction Prenatal depression is a serious maternal-child health concern; risk factors and health consequences appear more prevalent in Aboriginal communities and ethnic minority groups. However, research on these populations is limited. We examined the following questions: A) How do pregnant Aboriginal women, ethnic minority women and White/Caucasian women compare on levels of depressive symptoms, and on major risk and protective factors? B) Is non-dominant race associated with higher depressive symptoms? C) Through what pathways may race operate to contribute to depressive symptoms?

Methods Data were from the All Our Babies study $(\mathrm{n}=3354$ pregnant women from Alberta recruited between 2008-2011). Depressive symptoms were measured with the Edinburgh Postnatal Depression Scale (EPDS). Descriptive statistics and multivariable regression methods were used to assess the following hypotheses: We hypothesized that Aboriginal and ethnic minority women would have significantly higher mean EPDS score estimates relative to White/Caucasian women. The association between race and EPDS score was hypothesized to be partially mediated by risk factors such as socioeconomic factors, health background factors, discrimination, domestic violence, and psychosocial stress. Potential confounders were age, marital status, and parity. Diet and social support were hypothesized as protective buffers between stress and depressive symptoms.

Results White/Caucasian women appeared to have higher income, better employment and higher social support, and have significantly lower depressive symptoms. The association between race and depressive symptoms appeared partially mediated by socioeconomic factors and psychosocial stress; the attenuation with addition of stress in the model was particularly striking. However, race remained highly statistically significant as a predictor. Social support appeared to moderate the association between stress and depressive symptoms. Conclusion Mental health disparities between pregnant White/ Caucasian women and other women are driven by social disparities. A better understanding of the determinants of prenatal depression in specific populations may facilitate more effective public health and clinical interventions.

\section{PSYCHOSOCIAL STRESS PARTIALLY MEDIATES THE ASSOCIATION BETWEEN RACE AND PRENATAL DEPRESSIVE SYMPTOMS IN A SAMPLE OF PREGNANT WOMEN IN ALBERTA}

A Roy, ${ }^{1}$ SB Patten, ${ }^{1,2}$ WE Thurston, ${ }^{1,3}$ S Tough ${ }^{1,4}$. 'Department of Community Health Science, Faculty of Medicine, University of Calgary; ${ }^{2}$ Department of Psychiatry, Faculty of Medicine, University of Calgary; ${ }^{3}$ Department of Ecosystem and 